\title{
The Effect of Prolonged Lockdown Due to COVID-19 on Greek Demented Patients of Different Stages and on Their Caregivers
}

\author{
Angeliki Tsapanou ${ }^{\mathrm{a}, \mathrm{b}, *}$, Panagiota Zoi ${ }^{\mathrm{a}}$, Faidra Kalligerou ${ }^{\mathrm{a}}$, Patra Blekou ${ }^{\mathrm{a}}$ and Paraskevi Sakka ${ }^{\mathrm{a}}$ \\ a'Athens Alzheimer's Association, Athens, Greece \\ ${ }^{\mathrm{b}}$ Cognitive Neuroscience Division, Columbia University, New York, NY, USA
}

Accepted 3 July 2021

Pre-press 20 July 2021

\begin{abstract}
.
Background: The impact of the new coronavirus disease (COVID-19) is deteriorating as time passes and the virus keeps spreading, with people with dementia and their caregivers being affected significantly.

Objective: The aim of this study was to examine the effect of prolonged isolation because of the COVID-19 pandemic on people with dementia and their caregivers.

Methods: Caregivers answered online questions regarding their own physical and psychological burden, and of the person they take care of. Participants were mostly members of online seminars of the Athens Alzheimer's Association. Questions referred to their own burden, the overall decline of the persons they take care of, and changes in specific domains as well. Further, participants were asked about any changes between the two major lockdown periods. Analysis was performed including the total sample and then, by three different stages of dementia.

Results: A total of 339 caregivers took part in the study. Results indicated significant decline, both in an overall aspect of the people with dementia, and in specific domains (mostly communication and mood). Regarding the caregivers, they reported having significantly increased physical and psychological burden, and also, noticing an overall change between the two lockdown periods in their own burden. Analysis by dementia-stage group indicated that significant decline occurred both in the middle-stage and the late-stage group.

Conclusion: An urgency for further support of both the people with neurodegenerative disorders and their caregivers is needed. Collaboration among care workers, online programs, governmental support, and day-care centers should be planned to ensure continuity of care for those in need during the pandemic.
\end{abstract}

Keywords: Caregivers, COVID-19, dementia

\section{INTRODUCTION}

The ongoing new coronavirus disease (COVID19) has a profound impact on people of all ages and clinical symptoms, especially those suffering from neurodegenerative ailments. Prolonged periods of self-isolation creates increased feelings of

\footnotetext{
${ }^{*}$ Correspondence to: Angeliki Tsapanou, MSc, PhD, Clinical Neuropsychologist, Athens Alzheimer's Association, Cognitive Neuroscience Division, Columbia University, 622 W 168th st., New York, NY, 10032, USA. Tel.: +1 2034350545; E-mail: at2859@cumc.columbia.edu.
}

loneliness, behavioral modifications and disruptions, and acute events (i.e., agitation) requiring therapeutic changes or even hospitalization [1]. Especially regarding the restrictions having been put in many European countries, the situation affects significantly people suffering from dementia and their caregivers.

Although scarce, existing literature suggests that the COVID-19 outbreak is indirectly affecting the clinical conditions of people living with dementia and other related cognitive disorders [2]. The multifaceted health needs of the specific clinical group, unfortunately, were neglected in the emergency phase 
of the pandemic, rendering them even more challenging. Greece, after its economic crisis, was hit by another crisis - the pandemic, which had a dramatic negative impact not only in the economy but in population's life in total, especially considering that the country has the fourth lowest rate of health personnel employed in hospitals [3, 4]. Currently, there is a total of 243 care homes for elderly people operating in Greece. They cater to a total 15,000 elderly people, with about 10,000 staying in private facilities. Furthermore, there are currently no comprehensive universal formal long-term care services in Greece. Existing services are addressed to the neediest, poorest people. As mentioned by the EuroCarers organization, care for the chronically ill in Greece hardly covers the demand due to an insufficient number of beds, the low rates paid by social insurance organizations, and a rapidly shrinking public budget. Greece continues to suffer from a lack of a clearly formulated strategy and policies regarding the regulation of informal care and the support of informal caregivers, who amount to $34 \%$ of the Greek population [5-10]. As recommended by international dementia experts and the Alzheimer's Disease International [11], support for people living with dementia and their caregivers is urgently needed worldwide. In addition to physical protection from virus infection, mental health and psychosocial support should be delivered [12].

Regarding the caregivers of older adults, results of a recent study suggests a significantly increased overall burden and caregiving intensity [13]. The increased caring burden was predominant in women caregivers, even after adjusting for multiple covariates including caring intensity. A different study points out that dementia caregivers' perceptions about the impact of the pandemic (positive, concerned, irrelevant) varies, resulting in differential effects on their well-being [14]. The current pandemic seems to have increased the stress level of dementia caregivers, independently of the dementia stage, in an analysis of an Argentinian group. In the same study, half of the people suffering from dementia experienced increased anxiety, while most family members discontinued all of the cognitive and the physical therapies [15].

Our previous study showed a significant overall and by domain decline of the people with mild cognitive impairment/dementia [16]. Further, caregivers reported a significant increase in their psychological and physical burden during this period. In the current study, regarding the effect of the COVID-19 pandemic in patients with dementia and their caregivers, we aimed to 1 ) replicate the previous results in a much larger sample size, 2) examine the effect of the prolonged period of the lockdown (counting more than a year), 3) examine any changes between the two major phases of the lockdown (the first one took place during March to May 2020, while the second one covered November 2020-April 2021), and 4) expand on examining any differences based on the dementia stage of the person being taken care of.

\section{METHODS}

Caregivers answered an online questionnaire that we created regarding both changes of the patient they take care of, and changes to their own burden. Participants were from across Greece, being recruited by online seminars for caregivers that the Athens Alzheimer's Association organizes. The study took place from November 2020 to April 2021. Responses referred to the effect of the prolonged lockdown occurring in Greece, with possible answers: "not at all", "moderately", "a lot", and any change they noticed between the two major lock-down periods, with possible answers: "better", "the same", "worse". Questions referred to overall decline and in specific domains as well (mood, appetite, communication, compliance etc.). Informed consent was signed online prior to the completion of the questionnaire. Detailed information about the questions asked are provided in the Supplementary Material (in English and the original form, in Greek). Dementia stage: Dementia stage was self-reported, based, however, on the clinical diagnosis of the neurologist-provider.

\section{Statistical analysis}

For the analyses we used the Statistical Package for the Social Sciences (SPSS) 26 (SPSS, Chicago, Illinois). Characteristics of people with dementia and their caregivers were analyzed with descriptive (mean, standard deviation, frequency, and percent) and inferential statistics (chi-square test). Nominally significant alpha values were defined as $p<0.05$. Initial analysis performed in the total sample and then, we further created and analyzed three different stages of dementia (early, middle, late).

\section{RESULTS}

A total of 339 caregivers took part in the study (Fig. 1), with a mean age of 53 (S.D.: 13.9) years old. 


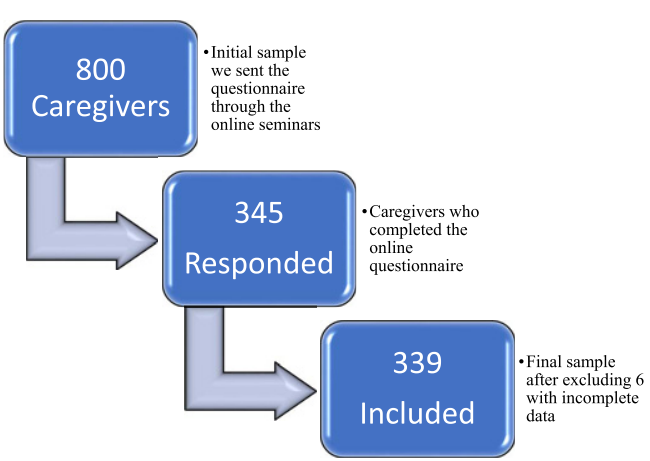

Fig. 1. Participants' chart.

People suffering from dementia were mostly women, with a mean age of 81 (S.D.: 7.5) years old, mostly being in the middle stage of dementia (52.2\%). Further details about the characteristics of patients and caregivers are provided in Table 1 . In order to examine the internal consistency of the items used in the analysis (Supplementary Material, questions 1-4, 15 items), we performed Cronbach's alpha statistical analysis, where results revealed a good reliability, with $a=0.792$ (for the purposes of the analysis, "Compliance with the new measures" was used with a reversed score, so that all questions indicate the same direction, higher score is worse).

Results indicated significant decline, both in an overall aspect of the people with dementia, and in specific domains. A percentage of $86.4 \%$ reported either moderate or a lot of overall change in the person with dementia (Fig. 2). Regarding the specific
Table 1

Characteristics of our sample

\begin{tabular}{lc}
\hline Characteristics & \\
\hline Sex (of the person with dementia) & \\
Women, $N(\%)$ & $217(64)$ \\
Sex (of the caregiver) & $206(85.8)$ \\
Women, $N(\%)$ & \\
Age of the patient, y, Mean (SD) & $81(7.5)$ \\
Age of the caregiver, y, Mean (SD) & $53(13.9)$ \\
Dementia Stage, $N(\%)$ & \\
$\quad$ Early & $79(23.3)$ \\
Middle & $177(52.2)$ \\
Late & $83(24.5)$ \\
Years of diagnosis, y, Mean (SD) & $4.7(3.2)$ \\
Total, $N$ & $\mathbf{3 3 9}$ \\
\end{tabular}

domains affected, most respondents reported a lot of change in the following: communication $(41.6 \%$ "a lot"), mood (37.5\% “a lot"), and mobility (33.3\% “a lot") (Table 2). The least change occurred for: delirium (52.5\% "not at all"), fear of going out (51.2\% "not at all"), and worries (48.1\% "not at all"). The difference also between the two lock-down periods seemed to be indicative, with $97 \%$ reporting the situation regarding the person with dementia during the second lock-down period being declined either "moderately" or "a lot" than the first one (Fig. 2). Qualitative data also showed that the vast majority of the support was coming from the Athens Alzheimer's Association online support services.

Regarding the caregivers, in the prolonged isolation due to the pandemic, they reported having significantly increased physical burden $(39.5 \%$ "a

Table 2

Changes in people suffering from dementia and their caregivers

\begin{tabular}{lccc}
\hline Question & Not at all & Moderate & A lot \\
& $N(\%)$ & $N(\%)$ & $N(\%)$ \\
\hline Overall decline & $46(13.6)$ & $204(60.2)$ & $89(26.3)$ \\
Physical health & $87(25.7)$ & $194(57.2)$ & $58(17.1)$ \\
Mobility & $58(17.1)$ & $168(49.6)$ & $113(33.3)$ \\
Mood & $39(11.5)$ & $173(51)$ & $127(37.5)$ \\
Appetite & $139(41)$ & $133(39.2)$ & $67(19.8)$ \\
Communication & $54(15.9)$ & $144(42.5)$ & $141(41.6)$ \\
Worries & $163(48.1)$ & $135(39.8)$ & $41(12.1)$ \\
Apathy & $87(25.7)$ & $161(47.5)$ & $91(26.8)$ \\
Wandering & $158(46.6)$ & $121(35.7)$ & $60(17.7)$ \\
Agitation & $95(28)$ & $158(46.6)$ & $86(25.4)$ \\
Delirium & $178(52.5)$ & $116(34.2)$ & $45(13.3)$ \\
Fear of going out & $177(51.2)$ & $109(2.2)$ & $53(15.6)$ \\
Compliance & $74(21.8)$ & $146(43.1)$ & $119(35.1)$ \\
Caregiver's physical burden & $51(15)$ & $154(45.4)$ & $134(39.5)$ \\
Caregiver's psychological burden & $33(9.7)$ & $101(29.8)$ & $205(60.5)$ \\
Change between lockdowns & $10(3)$ Better & $191(56.5)$ The same & $137(40.5)$ Worse \\
Caregiver's change & $14(4.1)$ & $130(38.5)$ & $194(57.4)$ \\
$\quad$ physical/psychological) & & & \\
between the lockdowns & & & \\
Total & $\mathbf{3 3 9}$ & &
\end{tabular}




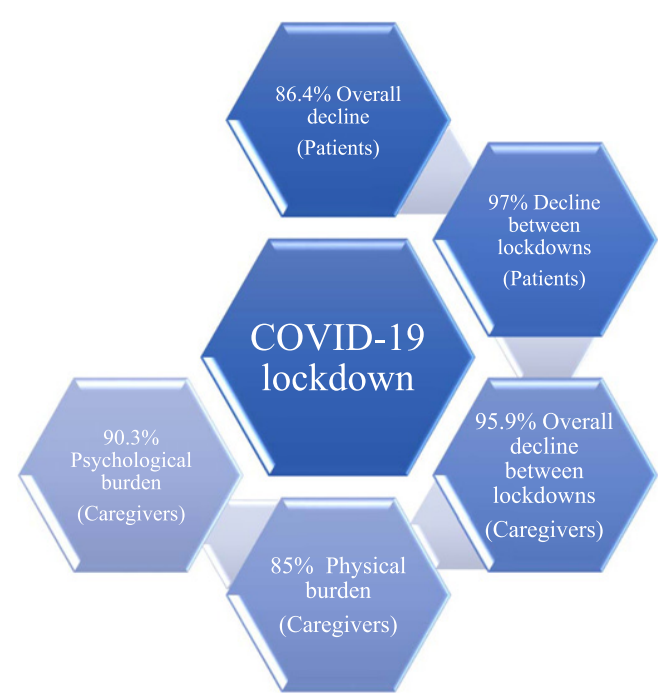

Fig. 2. Main changes (decline) for people with dementia and their caregivers, with "moderate" and "a lot" answers combined.

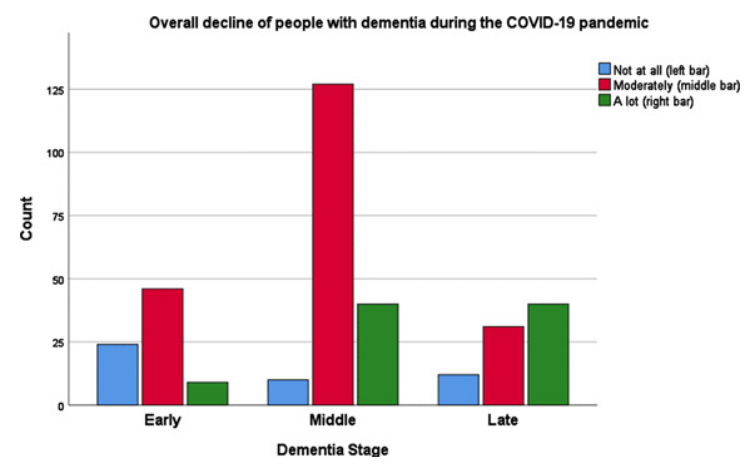

Fig. 3. Overall decline of people suffering from dementia during the COVID-19 pandemic, in three different stages of dementia.

lot", 85\% "moderately" and "a lot" combined). Most notable was the change in their psychology, with $60.5 \%$ reporting "a lot" of increase in their psychological burden. Combining the "moderately" and "a lot" answers, the proportion of the increased psychological burden of the caregivers during the prolong lockdown reached $90.3 \%$ (Fig. 2). Lastly, there was also a statistically significant change from the first to the second lock-down period both in their physical and their psychological burden (57.4\% "a lot", 95.9\% either "moderately" or "a lot") (Table 2, Fig. 2).

Analysis by dementia-stage group indicated that, interestingly, most of the "moderate" change was noticed for the middle-stage dementia patients, while the majority of change ("a lot") was reported for the late-stage dementia group (Table 3). Comparison among stage-groups showed significant associations

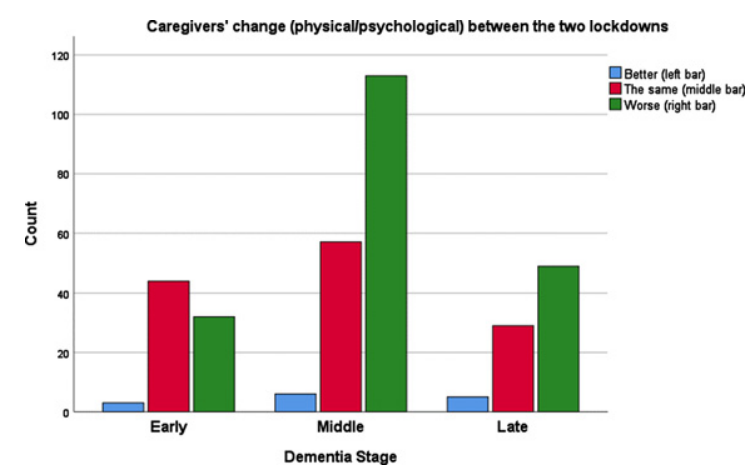

Fig. 4. Caregiver's change (physical/psychological) between the two lockdowns because of the COVID-19 pandemic, by dementia stage of the person they take care of.

for all the domains asked, except appetite and the change between the two lockdowns (Table 4). Figures 3, 4 show the change for the overall health/ behavior of the patient with dementia, and the caregiver's burden during the pandemic, by dementia stage.

\section{DISCUSSION}

With the enforced isolation due to the COVID19 pandemic, people suffering from dementia and their caregivers were highly affected, with statistically significant changes occurring in their total life. Our results are in accordance with previous reports of increased caregivers' burden and worsening of symptoms of people with neurodegenerative conditions [17]. We expanded our research on examining the impact by dementia-stage, where patients from all three stages seemed to be affected, especially in the domains of: physical health, mobility, and mood. Mostly, caregivers had support from the online programs of the Athens Alzheimer's Association, similarly to other data where internet and the prime physician of the day-care center were the predominant help for people with neurodegenerative conditions and their families [18].

The most significant effect occurred for the psychological burden of the caregivers, where, independently of the dementia stage of the person they take care of, they all expressed a lot of change in this domain. Thus, it seems that apart from the objective obstacles that caregivers have to face in the everyday life with a person with dementia (mood changes, personal hygiene, feeding, etc.), what affects them more is their own increased psychological burden. This 
Table 3

Changes by dementia-stage group

\begin{tabular}{|c|c|c|c|c|c|c|c|c|c|}
\hline & \multicolumn{3}{|c|}{ Early } & \multicolumn{3}{|c|}{ Middle } & \multicolumn{3}{|c|}{ Late } \\
\hline & $\begin{array}{c}\text { Not at all } \\
N(\%)\end{array}$ & $\begin{array}{l}\text { Moderate } \\
\quad N(\%)\end{array}$ & $\begin{array}{l}\text { A lot } \\
N(\%)\end{array}$ & $\begin{array}{l}\text { Not at all } \\
N(\%)\end{array}$ & $\begin{array}{l}\text { Moderate } \\
N(\%)\end{array}$ & $\begin{array}{l}\text { A lot } \\
N(\%)\end{array}$ & $\begin{array}{l}\text { Not at all } \\
N(\%)\end{array}$ & $\begin{array}{l}\text { Moderate } \\
N(\%)\end{array}$ & $\begin{array}{l}\text { A lot } \\
N(\%)\end{array}$ \\
\hline Overall decline & $24(30.4)$ & $46(58.2)$ & $9(11.4)$ & $10(5.6)$ & $127(71.8)$ & $40(22.6)$ & $12(14.5)$ & $31(37.3)$ & $40(48.2)$ \\
\hline Physical health & $35(44.3)$ & $38(48.1)$ & $6(7.6)$ & $36(20.3)$ & $115(65)$ & $26(14.7)$ & $16(19.3)$ & $41(49.4)$ & $26(31.3)$ \\
\hline Mobility & $29(36.7)$ & $39(49.4)$ & $11(13.9)$ & 17 (9.6) & $101(57.1)$ & $59(33.3)$ & $12(14.5)$ & $28(33.7)$ & $43(51.8)$ \\
\hline Mood & $13(16.5)$ & $50(63.3)$ & $16(20.3)$ & $13(7.3)$ & $94(53.1)$ & $70(39.5)$ & $13(15.7)$ & $29(34.9)$ & $41(49.4)$ \\
\hline Appetite & $42(53.2)$ & $27(34.2)$ & $10(12.7)$ & $65(36.7)$ & $74(41.8)$ & $38(21.5)$ & $32(38.6)$ & $32(38.6)$ & 19 (22.9) \\
\hline Communication & $26(32.9)$ & $38(48.1)$ & $15(19)$ & 21 (11.9) & $88(49.7)$ & $68(38.4)$ & $7(8.4)$ & $18(21.7)$ & $58(69.9)$ \\
\hline Worries & $14(17.7)$ & $49(62)$ & $16(20.3)$ & $83(46.9)$ & $73(4$ & $21(11.9)$ & $66(79.5)$ & $13(15.7)$ & $4(4.8)$ \\
\hline Apathy & $33(41.8)$ & $36(45.6)$ & $10(12.7)$ & $36(19.8)$ & $94(53.1)$ & 47 (26.6) & $18(21.7)$ & $31(37.3)$ & $34(41)$ \\
\hline Wandering & $46(58.2)$ & $26(32.9)$ & $7(8.9)$ & $75(42.4)$ & $72(40.7)$ & $30(16.9)$ & 37 (44.6) & $23(27.7)$ & $23(27.7)$ \\
\hline Agitation & $28(35.4)$ & $39(49.4)$ & $12(15.2)$ & $44(24.9)$ & $88(49.7)$ & $45(25.4)$ & $23(27.7)$ & $31(37.3)$ & $29(34.9)$ \\
\hline Delirium & $57(72.2)$ & $19(24.1)$ & $3(3.8)$ & $84(47.5)$ & $72(40.7)$ & $21(11.9)$ & 37 (44.6) & $25(30.1)$ & $21(25.3)$ \\
\hline Fear of going out & $36(45.6)$ & $32(40.5)$ & $11(13.9)$ & $93(52.5)$ & $62(35)$ & $22(12.4)$ & $48(57.8)$ & $15(18.1)$ & $20(24.1)$ \\
\hline Compliance & & & $40(50.6)$ & $32(18.1)$ & 88 & $57(32.2)$ & 37 & 24( & $22(26.5)$ \\
\hline Caregiver's physical burden & $21(26.6)$ & $43(54.4)$ & $15(19)$ & $23(13)$ & $80(45.2)$ & $74(41.8)$ & $7(8.4)$ & $31(37.3)$ & $45(54.2)$ \\
\hline $\begin{array}{l}\text { Caregiver's psychological } \\
\text { burden }\end{array}$ & $14(17.7)$ & $30(38)$ & $35(44.3)$ & $12(6.8)$ & $49(27.7)$ & $116(65.5)$ & $7(8.4)$ & $22(26.5)$ & $54(65.1)$ \\
\hline $\begin{array}{r}\text { Change between lockdowns } \\
\text { (Better, The same, Worse) }\end{array}$ & $4(5.1)$ & $48(60.8)$ & $27(34.2)$ & $6(3.4)$ & $90(51.1)$ & $80(45.5)$ & $0(0)$ & $53(63.9)$ & $30(36.1)$ \\
\hline $\begin{array}{l}\text { Caregiver's change } \\
\text { (physical/psychological) } \\
\text { between the lockdowns }\end{array}$ & $3(3.8)$ & $44(55.7)$ & $32(40.5)$ & $6(3.4)$ & $57(32.4)$ & $113(64.2)$ & $5(6)$ & $29(34.9)$ & $49(59)$ \\
\hline Total & & 79 & & & 177 & & & 83 & \\
\hline
\end{tabular}

burden was even more apparent in the prolonged lockdown period than the first one which occurred for only three months. In general, Greek population shares common cultural characteristics, and based on existing literature, people in Greece totally complied with the measures of social distancing during the COVID19 period $[19,20]$. Thus, they were highly socially and psychologically affected, especially regarding the feeling of isolation. Further, in the Greek society, social congregating for religious reasons around the Greek Orthodox church has an important element for social life that transcends spiritual needs. Considering this, the impact of social restriction had an even more severe effect in both the people with dementia and, subsequently, in their caregivers. Such reports indicate an urgent need for supporting the caregivers of people with neurodegenerative conditions. Further, our results are also following previous publication regarding the sex of the caregivers. Women are more likely than men to be informal caregivers, and among them, women are more likely to provide higher intensity care and experience greater burden than their male counterparts [13]. Similarly, in our study, the vast majority of the caregivers were women $(85.8 \%$ ), with $64 \%$ of the person taking care of being also of the same sex.

The current study, to our knowledge, is the first including both people with dementia in different
Table 4

Comparison of the changes among the dementia-stage groups

\begin{tabular}{lc}
\hline Question & $\chi^{2}, p$ \\
\hline Overall decline & $58.623,<0.0001$ \\
Physical health & $32.454,<0.0001$ \\
Mobility & $47.537,<0.0001$ \\
Mood & $21.887,<0.0001$ \\
Appetite & $7.275,0.122$ \\
Communication & $56.386,<0.0001$ \\
Worries & $62.278,<0.0001$ \\
Apathy & $25.581,<0.0001$ \\
Wandering & $14.172,0.007$ \\
Agitation & $10.434,0.034$ \\
Delirium & $27.266,<0.0001$ \\
Fear of going out & $13.573,0.009$ \\
Compliance & $42.789,<0.0001$ \\
Caregiver's physical burden & $25.695,<0.0001$ \\
Caregiver's psychological burden & $13.704,0.008$ \\
Change between lockdowns & $7.937,0.094$ \\
Caregiver's change & $14.405,0.006$ \\
$\quad$ (physical/psychological) & \\
between the lockdowns & \\
\hline
\end{tabular}

stages and their caregivers, regarding the effect of the prolonged lockdown, and with further evaluation of any changes between the two lockdown periods. However, some limitations should be also noted. The data are coming from self-reports, and no validated instruments to measure variables such as the physical or psychological burden were used. We also included a relatively small sample size. Further longitudi- 
nal evaluation, using objective measures is needed to examine the long-term consequences of the pandemic. Added to that, the lack of qualitative measures from two time-points regarding especially the burden of the caregivers is another limitation of the study.

Since all the population in quarantine has to deal with specific stressors both during the isolation (duration of quarantine, fears of infection, frustration, boredom, inadequate supplies) and post-quarantine (finances, stigma), situations like this should be handled carefully, providing clear communication, ensuring basic supplies, and providing meaningful activities [21]. Similarly to reports regarding other neurodegenerative conditions, i.e., Parkinson's disease, social isolation has been further compounded by the COVID-19 pandemic, emphasizing the need to keep older adults socially connected and prevent loneliness in this time of social distancing [22]. Other factors apart from isolation could also play a significant role to the worsening of symptoms of people with neurodegenerative conditions. The inability to use or the difficulty having access to new technology is a major factor, while increased mental strain and general mental health problems, and decreased exercise could have substantial negative effects on the elderly population [23].

The current hard situation indicates how imperative is to find solutions and design contingency plans for future crises, in order to guarantee properly sustained support to caregivers' increased burden [24]. As of 2021, the COVID-19 pandemic continues to impact nearly every aspect of living, and those impacts continue to evolve over time. Interviewing caregivers of people with dementia showed us the acute effects of the prolonged mandatory isolation in Greece. There is an urgent need for effective collaborations among public health programs, families, and health institutions in order to support caregivers and the people suffering from both a physical and a psychological aspect.

\section{DISCLOSURE STATEMENT}

Authors' disclosures available online (https:// www.j-alz.com/manuscript-disclosures/21-0702r2).

\section{SUPPLEMENTARY MATERIAL}

The supplementary material is available in the electronic version of this article: https://dx.doi.org/ 10.3233/JAD-210702.

\section{REFERENCES}

[1] Alzheimer Europe. COVID-19 - living with dementia. https://www.alzheimer-europe.org/Living-withdementia/COVID-19.

[2] Canevelli M, Valletta M, Toccaceli Blasi M, Remoli G, Sarti G, Nuti F, Sciancalepore F, Ruberti E, Cesari M, Bruno G (2020) Facing dementia during the COVID-19 outbreak. $J$ Am Geriatr Soc 68, 1673-1676.

[3] Giannopoulou I, Tsobanoglou GO (2020) COVID-19 pandemic: Challenges and opportunities for the Greek health care system. Ir J Psychol Med 37, 226-230.

[4] Economou C (2015) Barriers and Facilitating Factors in Access to Health Services in Greece. World Health Organization http://www.euro.who.int/en/countries/greece/ publications/barriers-and-facilitating-factors-in-access-tohealth-services-in-greece-2015.

[5] The 2018 Ageing Report, Economic and Budgetary Projections for the EU Member States (2016-2070), EC, 2018.

[6] ESPN Thematic Report on Challenges in long-term care Greece, EC, 2018.

[7] Informal care in Europe - Exploring Formalisation, Availability and Quality, LSE consulting, 2018.

[8] ESPN Thematic Report on work-life balance measures for persons of working age with dependent relatives, Greece, 2016.

[9] Joint Report on Health Care and Long-Term Care Systems and Fiscal Sustainability, EC, 2016.

[10] Adequate social protection for long-term care needs in an ageing society, European Commission, 2014.

[11] Alzheimer's Disease International. ADI offers advice and support during COVID-19. March 17, 2020. https:// www.alz.co.uk/news/adi-offers-advice-and-support-during -covid-19.

[12] Wang H, Li T, Barbarino P, Gauthier S, Brodaty H, Molinuevo JL, Xie H, Sun Y, Yu E, Tang Y, Weidner W, Yu X (2020) Dementia care during COVID-19. Lancet 395, 1190-1191.

[13] Cohen SA, Kunicki ZJ, Drohan MM, Greaney ML (2021) Exploring changes in caregiver burden and caregiving intensity due to COVID-19. Gerontol Geriatr Med 7, 2333721421999279.

[14] Savla J, Roberto KA, Blieszner R, McCann BR, Hoyt E, Knight AL (2021) Dementia caregiving during the "stay-athome" phase of COVID-19 pandemic. J Gerontol B Psychol Sci Soc Sci 76, e241-e245.

[15] Cohen G, Russo MJ, Campos JA, Allegri RF (2020) Living with dementia: Increased level of caregiver stress in times of COVID-19. Int Psychogeriatr 32, 1377-1381.

[16] Tsapanou A, Papatriantafyllou JD, Yiannopoulou K, Sali D, Kalligerou F, Ntanasi E, Zoi P, Margioti E, Kamtsadeli V, Hatzopoulou M, Koustimpi M, Zagka A, Papageorgiou SG, Sakka P (2021) The impact of COVID-19 pandemic on people with mild cognitive impairment/dementia and on their caregivers. Int J Geriatr Psychiatry 36, 583-587.

[17] Rainero I, Bruni AC, Marra C, Cagnin A, Bonanni L, Cupidi C, Lagana V, Rubino E, Vacca A, Di Lorenzo R, Provero P, Isella V, Vanacore N, Agosta F, Appollonio I, Caffarra P, Busse C, Sambati R, Quaranta D, Guglielmi V, Logroscino G, Filippi M, Tedeschi G, Ferrarese C, Group SIC-S (2020) The impact of COVID-19 quarantine on patients with dementia and family caregivers: A nation-wide survey. Front Aging Neurosci 12, 625781.

[18] Prouskas C (2020) Governmental response to the COVID19 pandemic in long-term care residences for older 
people: Preparedness, responses and challenges for the future: Greece. MC COVID-19 working paper 04/2021. http://dx.doi.org/10.20350/digitalCSIC/13695.

[19] Anastasiou E, Duquenne M-N (2021) What about the "Social Aspect of COVID"? Exploring the determinants of social isolation on the Greek population during the COVID19 lockdown. Soc Sci 10, 27.

[20] Parlapani E, Holeva V, Voitsidis P, Blekas A, Gliatas I, Porfyri GN, Golemis A, Papadopoulou K, Dimitriadou A, Chatzigeorgiou AF, Bairachtari V, Patsiala S, Skoupra M, Papigkioti K, Kafetzopoulou C, Diakogiannis I (2020) Psychological and behavioral responses to the COVID-19 pandemic in Greece. Front Psychiatry 11, 821.

[21] Brooks SK, Webster RK, Smith LE, Woodland L, Wessely S, Greenberg N, Rubin GJ (2020) The psychological impact of quarantine and how to reduce it: Rapid review of the evidence. Lancet 395, 912-920.
[22] Subramanian I, Farahnik J, Mischley LK (2020) Synergy of pandemics-social isolation is associated with worsened Parkinson severity and quality of life. NPJ Parkinsons Dis 6, 28.

[23] Martins Van Jaarsveld G (2020) The effects of COVID-19 among the elderly population: A case for closing the digital divide. Front Psychiatry 11, 577427.

[24] Borges-Machado F, Barros D, Ribeiro O, Carvalho J (2020) The effects of COVID-19 home confinement in dementia care: Physical and cognitive decline, severe neuropsychiatric symptoms and increased caregiving burden. Am J Alzheimers Dis Other Demen 35, 1533317520976720. 